Positions Available

\section{PROCESS \\ DEVELOPMENT \\ ENGINEER}

KEMIRA is an international chemicals manufacturer and the fifth largest producer of Titanium Dioxide pigments. We are proud of our advanced technology, environmental control, team-concept manufacturing, and aggressive marketing. Currently, we have an opening for a professional with an MS/PhD degree in Chemical Engineering as well as research experience in aerosols or fast high temperature reactions.

The successful candidate will be responsible for Process Development work on our TiO2 pigment manufacturing process, studies of thermochemical equilibria and kinetics and model development and simulation.

Along with technical challenge and diversity, we offer competitive compensation, full benefits and professional recognition. Qualified Engineers are invited to submit resume, cover letter and salary expectations to: Personnel Manager, Kemira, Inc., P.O. Box 368, Savannah, GA 31402. Equal opportunity employer.

\section{Positions Wanted}

Postdoc in materials science and engineering seeks position in industrial/academic R\&D in processing development and characterization of materials; PhD in solid-state physics with several years of diversified experience in materials science. Background in crystalline theory, solidstate physics, electronic materials processing, and computer modeling and simulation. Instrumentation: conventional and high-resolution TEM, including JOEL, Philips and Topcon EM; high-spatial-resolution spectroscopies (EELS and EDXS); CBED, SEM, and x-ray diffraction. Please reply to Box XVIII, No. 602.

Industrial/Academic Postdoctoral position: $\mathrm{PhD}$ in ceramic science. Three years experience in refractories manufacturing and development. Research-chemical processing of $\mathrm{BaTiO}_{3}$ films and powders, crystallization in $\mathrm{Y}_{2} \mathrm{O}_{3}-\mathrm{SiO}_{2}$ glasses. Characterization skills-TEM, SEM, XRD, DTA, TGA, FT- IR, size, density, and surface area. Excellent communication and interpersonal skills. Please reply to Box XVIII, No. 601 .

\section{Employers-}

TO REPLY TO BOX NUMBER, WRITE:

Box_, No.__ c/o MRS Bulletin

Materials Research Society

9800 McKnight Road, Pittsburgh, PA 15237

\section{RESEARCH ASSOCIATE}

Norian, a growing Class III medical device company, is seeking a research associate for biocompatible cement research. Responsibilities include cement formulation and development, mechanical and analytical characterization (e.g., Instron, XRD, FTIR, UV/vis., BET, particle size, etc.), equipment maintenance and calibration, and general laboratory duties. BS in materials engineering (or equivalent), with 1+ years laboratory experience required. Should be computer literate and have strong mechanical aptitude. Please send resume to: Norian Corporation, Attn. MF and II, 1025 Terra Bella Ave., Mountain View, CA 94043, or fax to (415) 968-5742.

Ad closing for the September MRS Bulletin is

\section{August 1, 1993}

To place your ad, call Mary E. Kaufold at (412) 367-3036 today!

\title{
BriefCommunication* The Effect of Toxic Microbes on Accelerator Performance
}

I.M. Smart, Exochemistry Department, I.C. University

U.R. Tu, Materials Science Publishing Unit, P.R. First, Inc.

and

B.A. Sport, Society for the Prevention of Vacuum in Outer Space

\section{ABSTRACT}

The potential of the environment inside evacuated accelerator beamlines for breeding microbes which are both toxic to users and possess electric or magnetic organs of strength sufficient to deflect soft chargedparticle beams has been explored. Unexplained human and accelerator "long-runtime" syndromes plaguing accelerator facilities for many years can be understood from this study. We have artificially bred such organisms and demonstrated that they are the cause of these syndromes. An appropriate microbicide-impregnated antiseptic beampipe liner with good outgassing properties could alleviate the problem.

Introduction

Anecdotal evidence from experiences of accelerator physicists regarding the contemporaneous occurrence of flu symptoms (including delusionary episodes) and unexplained beam-optics and transmission problems after long 
(>24 hours) continuous experimental runs has accumulated over the years. ${ }^{1}$ These phenomena have conventionally been attributed to fatigue and operator error. However, they could be explained by the existence of vacuum-resistant microbes that are toxic or infectious and display an electric or magnetic "charge.."

Our study has involved attempts to artificially culture such microbes in simulated accelerator environments. Once adequate quantities of suspected microbes were obtained, their toxicity and electromagnetic properties were evaluated.

\section{Experimental Procedure}

After construction of an accelerator incubation chamber and associated lab facilities for breeding experiments, one year was devoted to monitoring and recording the growth, if any, of 100,000 test specimens obtained from a wide variety of typical accelerator components and materials. Cultures utilized a silicone pump-oil medium and were exposed aperiodically to irradiation by $x$-rays in the one to one-hundred $\mathrm{keV}$ range.

The toxicity of the candidate organisms, grown as above, was then tested in standard clinical trials using volunteer accelerator physicists who were exposed to the microbes after working for 24 hours without sleep. Exposure was accomplished through inhalation of airborne samples, ingestion while eating typical accelerator-lab fare, or absorption through pores from a vacuum grease medium. A control subgroup of subjects was exposed to a specially developed "placebo" bacterium which has no known effect on humans, in general, or physicists, in particular.

Free fall through charged parallel plates or, alternatively, through a gap between poles of permanent magnets was used to infer mass and electromagnetic properties. No corrections for aerodynamic profile of the microbes were needed as the tests were conducted under a vacuum of $10^{-10}$ torr (neglecting any microbial outgassing). However, a special self-stabilizing and lateral momentum compensating microbe-release shoot had to be devised to account for a random lateral impulse observed to affect microbe trajectories. Initial trials showed only partial compensation; however, after painting the entry ends of the parallel plates a less threatening hue of pastel blue, the strength of the lateral "pushoffs" fell within the compensator's effective range.

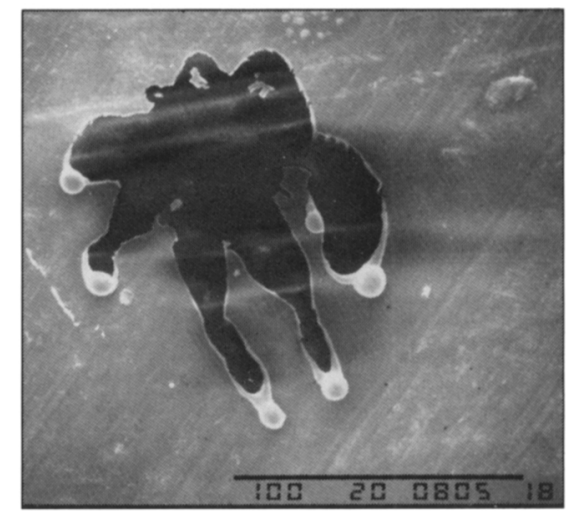

Replication ${ }^{* *}$ scanning electron micrograph of isolated organism believed to be the bacillus, electrovacuoacceleratidae.

\section{Results}

Cultures were successfully grown from a wide range of organically contaminated accelerator parts. Colonies of species that feed primarily on pump oil residues complexed with neoprene, stainless steel (chromium component in particular), and pyrolyzed carbon were the most prolific. However, even for these, incubation periods up to six months were required before the colonies showed self-sufficiency and could migrate at will via oxides and oxygen-bearing polymers. A theoretical simulation confirmed that, although radiation-induced life from cross polymerization of noncontaminated accelerator media had a substantial likelihood, the probability was not sufficient to account for the rash of reported accelerator-syndrome events.

Only one of the strains of cultured bacteria both induced flu symptoms in subjects and showed marked deflections when dropped in vacuum through the electric and magnetic fields. We can estimate an average object mass $=7.5 \times 10^{17}$ a.u. and average electric charge $=-730 e$. No net magnetic moment could be observed with our apparatus in the presence of the large electric charge. Interestingly, although this particular species only survives minutes outside a vacuum system, it is likely that it is responsible for the above-described syndrome.

\section{Conclusion}

The identified strain, electrovacuoacceleratidae, is clearly responsible for flu-like symptoms, but its electric charge is too small to directly deflect a particle beam of any rigidity. It is likely that reported "deflection" is a misinterpretation of loss of transmitted beam. Secondary electron emission from oxidized surfaces in and around collimator orifices can produce a positive charge that attracts colonies of the bacilli which partially block and/or degrade the beam.

We believe these findings will eventually result in a recall of all accelerator components manufactured after 1900 and which have been used in systems based on oil diffusion or oil-based roughing pumps and/or polymer rather than metal gaskets. In addition, it is likely that the Clean Vacuum Act of 1993 will require bakeable antimicrobial coatings on the inner surfaces of all accelerator beampipes by the year 2000 .

We are now turning our attention to developing a related hybrid bacterium that is electrically neutral and, at moderate exposure levels, will induce only the principal and most redeeming reported symptom, the so-called "midnight genius" syndrome.

\section{Acknowledgments}

Additional participants in this work include Prof. X.Y. Zee of the Alphabetic Recognition Institute, who monitored subjects' coherence during clinical tests, and O.G. Wiz, a postdoctoral fellow from the Dream Prevention Center, who studied correlations between fatigue, vacuum accident probability, and creative thought processes while maintaining subjects in their awake state.

*In order to insure rapid publication, the usual peer review process was skipped and only neatness of the original manuscript was considered.

* Rather than employing the conventional replicasample method for electron microscopy on insulating samples, we have used the replica-micrograph method, wherein one sifts through old files of unrelated micrographs until one finds one that resembles what one imagines one would have seen had the microscopy work actually been done. This figure was received by the MRS Bulletin in January 1989 from I. Kleiman of the Institute for Aerospace Studies of the University of Toronto, but had yet to rise to the top of the supply of figures for the Editor's Choice department. In actuality, it depicts a region of a gold-coated sample of pyrolitic graphite where the gold failed to adhere.

\section{References}

1. "The Elusive Magnetic Microbe and Other Accelerator Tales," I.M. Smart, U.R. Tu, and B.A. Sport, in Stainless Stecl and You, edited by E.Z. Judge (Vanity Press, Waxahachie, 1991) p. 2001-2010. (Reprints sold out.)

2. "Magnetic Microbes and the Common Cold," U.R Tu, I.M. Smart, and X.Y. Zee, Nezv England Journal of Magnetism 666 (1992) p. 7-11. (Reprints for sale by the authors. Get them while they last?) 